\title{
Prevalence and incidence of antimicrobial-resistant organisms among hospitalized inflammatory bowel disease patients
}

\author{
Alon Vaisman MD¹, Kevin Pivovarov HBSc${ }^{1}$, Allison McGeer MD², Barbara Willey ART², Bjug Borgundvaag MD FRCPC ${ }^{3}$, \\ Vanessa Porter $\mathrm{MLT}^{2}$, Piraveina Gnanasuntharam BSc${ }^{2}$, Yanliang Wei BSc ${ }^{2}$, Geoffrey C Nguyen MD PhD FRCPC ${ }^{1,4}$
}

\begin{abstract}
A Vaisman, K Pivovarov, A McGeer, et al. Prevalence and incidence of antimicrobial-resistant organisms among hospitalized inflammatory bowel disease patients. Can J Infect Dis Med Microbiol 2013;24(4):e117-e121.
\end{abstract}

BACKGROUND: Patients with inflammatory bowel disease (IBD) experience frequent hospitalizations and use of immunosuppressive medications, which may predispose them to colonization with antimicrobial-resistant organisms (ARO).

OBJECTIVE: To determine the prevalence of ARO colonization on admission to hospital and the incidence of infection during hospitalization among hospitalized IBD patients.

METHODS: A chart review comparing the prevalence of colonization and incidence of infection with methicillin-resistant Staphylococcus aureus, vancomycin-resistant enterococci and extended-spectrum betalactamase-producing Enterobacteriaceae (ESBL) in hospitalized IBD patients with those of non-IBD controls was performed.

RESULTS: On admission, there were no significant differences between IBD inpatients and controls in the prevalence of colonization of methicillin-resistant $S$ aureus (1.0\% versus $1.2 \% ; \mathrm{P}=0.74)$, vancomycinresistant enterococci $(0.2 \%$ versus $0 \% ; \mathrm{P}=1.0)$ or ESBL ( $4.1 \%$ versus $5.5 \% ; \mathrm{P}=0.33$ ). Pooling data from historical clinic-based cohorts, IBD patients were more likely than controls to have ESBL colonization (19\% versus $6.6 \% ; \mathrm{P}<0.05)$. Antibiotic use on admission was associated with ESBL colonization among IBD inpatients (OR 4.2 [95\% CI 1.4 to 12.6]). The incidence of $A R O$ infections during hospitalization was not significantly different between IBD patients and controls. Among IBD patients who acquired ARO infections during hospitalizations, the mean time interval from admission to infection was shorter for those who were already colonized with ARO on admission.

CONCLUSIONS: This particular population of hospitalized IBD patients was not shown to have a higher prevalence or incidence of ARO colonization or infection compared with non-IBD inpatients.

Key Words: Crohn disease; Extended spectrum beta-lactamase; Inflammatory bowel disease; Methicillin-resistant Staphylococcus aureus; Ulcerative colitis; Vancomycin-resistant enterococci
La prévalence et l'incidence d'organismes résistants aux antimicrobiens chez les patients hospitalisés atteints d'une maladie inflammatoire de l'intestin

HISTORIQUE : Les patients atteints d'une maladie inflammatoire de l'intestin (MII) sont souvent hospitalisés et prennent souvent des immunosuppresseurs, ce qui peut les prédisposer à des organismes résistants aux antimicrobiens (ORA).

OBJECTIF : Déterminer la prévalence de colonisation par des ORA à l'admission à l'hôpital ainsi que l'incidence d'infection pendant l'hospitalisation de patients atteints d'une MII.

MÉTHODOLOGIE : Les chercheurs ont procédé à l'analyse des dossiers comparant la prévalence de colonisation et d'incidence d'infection par le Staphylococcus aureus résistant à la méthicilline (SARM), les entérocoques résistant à la vancomycine (EVM) et les entérobactériacées productrices de bêta-lactamase à spectre étendu (BLSE) des patients hospitalisés atteints d'une MII à celle de sujets témoins n'ayant pas de MII.

RÉSULTATS : À l'admission, les chercheurs n'ont pas constaté de différences significatives entre les patients hospitalisés atteints d'une MII et les sujets témoins pour ce qui est de la prévalence de colonisation par le SARM (1,0 \% par rapport à $1,2 \% ; \mathrm{P}=0,74)$, les EVM $(0,2 \%$ par rapport à $0 \% ; \mathrm{P}=1,0)$ ou les $\mathrm{BLSE}(4,1 \%$ par rapport à $5,5 \% ; \mathrm{P}=0,33)$. Selon les données regroupées de cohortes cliniques rétrospectives, les patients atteints d'une MII étaient plus susceptibles que les sujets témoins d'être colonisés par des BLSE (19\% par rapport à 6,6 \%; $\mathrm{P}<0,05)$. L'utilisation d'antibiotiques à l'admission s'associait à une colonisation par les BLSE chez les patients atteints d'une MII (RRR 4,2 [95 \% IC1,4 à 12,6]). L'incidence d'infections par des ORA pendant l'hospitalisation n'était pas significativement différente entre les patients atteints d'une MII et les sujets témoins. Chez les patients atteints d'une MII qui avaient contracté une infection par des ORA pendant l'hospitalisation, l'intervalle moyen entre l'admission et l'infection était plus court pour ceux qui étaient déjà colonisés par des ORA à l'admission.

CONCLUSIONS : Cette population de patients hospitalisés atteints d'une MII ne présentait pas de prévalence ou d'incidence plus élevée de colonisation par des ORA que les patients n'ayant pas de MII.

increase costs, and increase morbidity and mortality. Screening programs for MRSA have been shown to have a positive cost-benefit ratio (3-5). The risk factors for acquisition of MRSA, VRE and ESBL infections are similar and include advanced age, underlying diseases, prolonged hospitalization, gastrointestinal surgery, exposure to invasive devices (especially central venous catheters) and exposure to antimicrobial drugs (6).

More than one-quarter of hospitalizations for inflammatory bowel disease (IBD), which comprises Crohn disease (CD) and ulcerative Colonization and infection with ARO prolong hospitalizations,

${ }^{1}$ Mount Sinai Centre for Inflammatory Bowel Disease, University of Toronto; ${ }^{2}$ Department of Microbiology, Mount Sinai Hospital, University of Toronto; ${ }^{3}$ Department of Emergency Medicine, Mount Sinai Hospital, University of Toronto, Toronto, Ontario; ${ }^{4}$ Johns Hopkins University School of Medicine, Baltimore, Maryland, USA

Correspondence: Dr Geoffrey C Nguyen, Mount Sinai Hospital, 600 University Avenue, Room 437, Toronto, Ontario M5G 1 X5.

Telephone 416-586-4800 ext 2819, fax 416-586-5971, e-mail geoff.nguyen@utoronto.ca 
colitis (UC), are associated with infections, including those by ARO. These infections are associated with fourfold higher mortality (7). These statistics become increasingly relevant as the number of hospitalizations for IBD in North America continue to rise (8). As many as $83 \%$ of individuals with CD are hospitalized within the first year of diagnosis, and an additional 20\% are hospitalized within each of the next four years (9). IBD patients are also more likely to undergo major surgery early during their disease, with $38 \%$ of $\mathrm{CD}$ patients requiring surgery within the first 10 years of diagnosis (10). Moreover, the frequent use of immunosuppressive therapy also renders IBD patients susceptible to serious infections $(7,11,12)$.

As a consequence of the above factors, hospitalized IBD patients may be at increased risk for acquiring several AROs. Patients with IBD have been consistently shown to experience increased rates of Clostridium difficile infection, with rates increasing dramatically in recent years $(13,14)$. Hospitalized IBD patients were also found to have increased risk of VRE and MRSA infection compared with nonIBD controls (15). MRSA infections, which more than tripled between 1998 and 2004 among hospitalized IBD patients, were associated with sevenfold higher in-hospital mortality (3). Finally, in one study, one-third of IBD patients who developed pouchitis following restorative proctocolectomy were colonized with ESBL (16).

It is unknown whether hospitalized IBD patients in Canada have a similar increased risk for MRSA infection to their American counterparts. Furthermore, it remains unclear whether IBD patients acquire MRSA infection de novo during hospitalization or whether they are admitted with MRSA colonization that predisposes them to subsequent infection. A Toronto (Ontario)-based study suggested that the prevalence of ARO colonization is similar between IBD patients and non-IBD controls in the outpatient setting (17). However, IBD patients who require hospitalization may reflect a different subgroup of patients with respect to disease severity and exposure to risk factors for ARO.

The aim of the present study was to characterize the prevalence of ARO colonization among hospitalized IBD patients on admission at a tertiary IBD centre. Furthermore, we sought to assess the risk of colonization and infection during hospitalization.

\section{METHODS}

\section{Patient selection criteria}

Medical records of all IBD patients admitted with a most responsible diagnosis of IBD to Mount Sinai Hospital (Toronto, Ontario) from January 1, 2008 to December 31, 2009 were identified and reviewed. Control patients were selected from the general internal medicine wards admitted from January 1, 2008 to December 31, 2009 at the same tertiary centre. Patients admitted due to an ARO infection were excluded from the analysis.

All patients included in the study underwent ARO screening with culture swabs sampled on admission as part of the standard protocol at the institution. Nasal swabs were collected for MRSA, and rectal swabs were collected for ESBL, MRSA and VRE. Culture results were collected from admission screening as well as additional clinically indicated blood, urine, sputum and/or tissue cultures. Demographic data and data on comorbid conditions, namely diabetes mellitus, HIV and chronic kidney disease, were collected for each patient from medical records.

Two additional historical control groups were included in the analysis (17): one group of IBD patients from the ambulatory clinics at Mount Sinai Hospital recruited between July 15, 2010 and December 21, 2010; and a non-IBD cohort from the centre's affiliated family practice clinic recruited between June 15 and August 15, 2008 that was recruited as part of another MRSA prevalence study. The ambulatory IBD control group was used a comparator to assess potential differences in MRSA risk between the subgroup of IBD patients who required hospitalization and the broader ambulatory IBD population. Subjects from the ambulatory clinics of the Mount Sinai Hospital family practice unit were screened for MRSA as part of a study protocol and not for any specific clinical indication. These patients were selected to represent the broader non-IBD patient population seen at the centre.

\section{Ethical considerations}

The present study was approved by the Research Ethics Board of Mount Sinai Hospital.

\section{Outcome variables}

Primary outcomes included: prevalence of colonization on admission to the hospital of MRSA, VRE or ESBL; and incidence of clinically defined infections by MRSA, VRE and ESBL. To determine risk factors for ARO acquisition, the following preadmission variables were extracted by chart review: previous admissions and/or recent ( $<3$ months) history of surgery, chemotherapy and/or antibiotic use. Additional in-hospital factors recorded were: surgical procedures including bowel surgery; use of invasive devices such as urinary catheters, central lines and ventilators; use of blood products, total parenteral nutrition (TPN) and/or antibiotics; duration of hospital stay; and admission to the intensive care unit.

\section{Statistical analyses}

Data were analyzed using the Stata 10.0 SE software package (StataCorp, USA). Fischer's exact test was used to compare baseline clinical characteristics and outcomes between hospitalized IBD patients and control groups. Logistic regression analysis was used to determine whether any of these factors were associated with ARO colonization on admission or development of infection during hospitalization.

\section{Cohort characteristics}

\section{RESULTS}

Patient characteristics of hospitalized IBD patients and control groups are outlined in Table 1. In total, the charts of 537 IBD patients were reviewed: 246 (54\%) with UC and 291 (46\%) with CD. Characteristics of IBD inpatients, including disease extent, previous surgery and disease activity, are listed in Table 1 and represent a typical IBD population. These clinical details were not available for the ambulatory IBD group. A total of 345 inpatient control patients met criteria for inclusion in the study. Both ambulatory and inpatient non-IBD control groups were older, on average, than their IBD counterparts.

\section{Prevalence of MRSA, ESBL and VRE}

The prevalence of ARO in the IBD and control groups are detailed in Table 2. Overall, there were no significant differences between the IBD and non-IBD inpatient groups in the prevalence of MRSA $(1.0 \%$ versus $1.2 \% ; \mathrm{P}=0.74)$, $\operatorname{VRE}(0.2 \%$ versus $0 \% ; \mathrm{P}=1.0)$ or $\operatorname{ESBL}(4.1 \%$ versus $5.5 \% ; \mathrm{P}=0.33)$. When data from both inpatient and clinic study populations were combined, IBD patients and non-IBD controls exhibited a similar prevalence of ESBL colonization (6.6\% versus $5.5 \% ; \mathrm{P}=0.5)$ and MRSA colonization ( $1.1 \%$ versus $1.1 \% ; \mathrm{P}=1.0)$, respectively. Compared with inpatients, IBD patients from the IBD clinics were more likely to have ESBL infection $(11.1 \%$ versus $4.1 \%$; $\mathrm{P}<0.05)$ but not MRSA infection $(1.6 \%$ versus $1.0 \% ; \mathrm{P}=0.51)$.

\section{Predictive factors for ARO}

The prevalences of previously described risk factors for ARO acquisition among IBD patients and non-IBD controls are listed in Table 3. Antibiotic use on admission was found to be associated with ESBL colonization on admission among those with IBD (OR 4.2 [95\% Cl 1.4 to 12.6$)$ ]. Chronic kidney disease was associated with MRSA colonization on admission (OR 16.1 [95\% CI 2.6 to 100.2]) only among non-IBD inpatients.

\section{Incidence of colonization/infection during admission}

Data regarding the incidence of colonization/infection during admission are presented in Table 4. Overall, patients with IBD were not more likely to acquire MRSA, ESBL or VRE infections or colonization during admission than those in the control group. A total of 31 IBD patients had positive samples either on admission or during admission. The time intervals to colonization or infection with $\mathrm{ARO}$ are detailed in Table 5. The average time to develop infection among patients who 
TABLE 1

Cohort characteristics of inpatients and outpatients of inflammatory bowel disease (IBD) and control groups

\begin{tabular}{|c|c|c|c|c|c|c|}
\hline \multirow[b]{2}{*}{ Characteristic } & \multicolumn{2}{|c|}{ Individual IBD groups } & \multirow[b]{2}{*}{ All IBD groups } & \multicolumn{2}{|c|}{ Individual control groups } & \multirow[b]{2}{*}{ All control groups } \\
\hline & Inpatients & Outpatients* & & Inpatients & Outpatients* (family clinic) & \\
\hline $\mathrm{n}$ & 537 & 306 & 843 & 345 & 190 & 535 \\
\hline Age, years, mean & 38 & 39 & 38 & 69 & 55 & 64 \\
\hline Female sex, $\%$ & 52 & 49 & 51 & 48 & - & 48 \\
\hline Presence of perianal disease & $65(22)$ & - & - & - & - & - \\
\hline \multicolumn{7}{|l|}{ Disease activity } \\
\hline Inflammatory & $122(42)$ & - & - & - & - & - \\
\hline Stricturing & $75(26)$ & - & - & - & - & - \\
\hline Penetrating & $94(32)$ & - & - & - & - & - \\
\hline \multicolumn{7}{|l|}{ Crohn disease $(n=291)$} \\
\hline \multicolumn{7}{|l|}{ Disease location } \\
\hline Colonic & $66(23)$ & - & - & - & - & - \\
\hline Ileal & $68(24)$ & - & - & - & - & - \\
\hline Ileocolonic & $142(49)$ & - & - & - & - & - \\
\hline Upper gastrointestinal tract & $15(5)$ & - & - & - & - & - \\
\hline Ulcerative colitis $(n=246)$ & & - & - & - & - & - \\
\hline \multicolumn{7}{|l|}{ Disease extent } \\
\hline Proctitis & $8(3.2)$ & - & - & - & - & - \\
\hline Left-sided & $113(46)$ & - & - & - & - & - \\
\hline Pancolitis & $125(51)$ & - & - & - & - & - \\
\hline
\end{tabular}

Data presented as $n$ (\%) unless otherwise indicated. *Data from reference 17

TABLE 2

Prevalence of antimicrobial-resistant organisms in inflammatory bowel disease (IBD) inpatients on admission and control groups

\begin{tabular}{|c|c|c|c|c|c|c|}
\hline & \multicolumn{2}{|c|}{ Individual IBD groups } & \multirow[b]{2}{*}{ All IBD groups } & \multicolumn{2}{|c|}{ Individual control groups } & \multirow[b]{2}{*}{ All control groups } \\
\hline & Inpatients & Outpatients & & Inpatients & Outpatients (family clinic) & \\
\hline $\mathrm{n}$ & 537 & 306 & 843 & 345 & 190 & 535 \\
\hline MRSA & $5(1.0)$ & $5(1.6)$ & $10(1.1)$ & $4(1.2)$ & $2(1.1)$ & $6(1.1)$ \\
\hline VRE & $1(0.2)$ & $0(0)$ & $1(0.1)$ & $0(0)$ & * & $0(0)$ \\
\hline ESBL & $22(4.1)$ & $34(11.1)^{\dagger}$ & $56(6.6)$ & $19(5.5)$ & * & $19(5.5)^{\ddagger}$ \\
\hline
\end{tabular}

Data presented as $n$ (\%) unless otherwise indicated. *Vancomycin-resistant Enterococcus species (VRE) and extended-spectrum beta-lactamase-producing Enterobacteriaceae (ESBL) swabs were not collected in the family clinic control group; ${ }^{\dagger} P<0.05$ compared with IBD inpatients; $\neq P<0.05$ compared with all IBD groups. MRSA Methicillin-resistant Staphylococcus aureus

were colonized with MRSA and ESBL on admission were 12.3 and seven days, respectively. For those not colonized with MRSA and ESBL on admission, the time intervals to infection were 20 and 16.5 days, respectively.

\section{DISCUSSION}

In our single-centre study, we observed that the prevalence of MRSA and ESBL colonization on admission did not significantly differ between hospitalized IBD patients and non-IBD inpatients. This finding is consistent with our previous study of ARO prevalence among IBD ambulatory clinic patients and non-IBD controls recruited from the family practice clinics and emergency departments from the same tertiary centre (17). These findings are reassuring given previous reports that IBD patients incur a greater number of health care contacts and hospitalizations than the general population (18).

When we combined the data from our inpatient IBD population with those of IBD patients seen in our tertiary care IBD clinic, we found that ESBL colonization was more frequent in the IBD population compared with non-IBD controls. Antibiotic use on admission was the only predictor of ESBL colonization on admission. In our study, antibiotic use on admission was higher among IBD inpatients compared with non-IBD inpatients and may have partly explained the higher ESBL colonization among IBD patients in general. These observations may also be explained by the frequent use of antibiotics in IBD for primary treatment of this inflammatory condition as well as its infectious complications.
The findings from our inpatient study showed no difference in colonization with MRSA on admission between IBD inpatients and non-IBD controls, confirming our previous study of IBD and non-IBD populations in the clinic setting (17). These observations are in contrast to nationwide hospital-based studies from the United States, which demonstrated an increased risk of MRSA infection among IBD inpatients compared with non-IBD controls (3). This discrepancy may be partly due to the overall low rates of $\mathrm{ARO}$ at our institution compared with nationwide averages in Canada, possibly reflecting effective infection prevention and control practices $(2,19)$. Furthermore, there may differences in basal colonization and infection control practices between Canada and the United States (20).

Our study further aimed to identify factors that increase the likelihood of ARO colonization. Chronic kidney disease, a well-established risk factor for ARO acquisition, was the only predictive factor for MRSA colonization in all patients, but not in IBD patients alone (21). Colonization with ESBL on admission was associated with antibiotic use on admission in our IBD patients, a finding consistent with previous studies $(16,17)$. However, previously established risk factors for $\mathrm{ARO}$ acquisition, including use of total parenteral nutrition, immunosuppressive drugs and recent bowel surgery were not associated with MRSA or ESBL colonization in our IBD population. This inconsistency may again be partly due to the focus on hand hygiene compliance and infection prevention, which are at the root of low rates of $\mathrm{ARO}$ acquisition at our centre. 
TABLE 3

Risk factors for antimicrobial-resistant organism acquisition

\begin{tabular}{lccc}
\hline & \multicolumn{2}{c}{ Inpatient group } & \\
\cline { 2 - 3 } Risk factor & $\begin{array}{c}\text { IBD } \\
\text { (n=537) }\end{array}$ & $\begin{array}{c}\text { Non-IBD } \\
(\mathbf{n}=\mathbf{3 4 5})\end{array}$ & $\mathbf{P}$ \\
\hline Preadmission factors & & & \\
$\quad$ History of surgery before admission & $252(47)$ & $117(34)$ & $<0.05$ \\
Antibiotics 3 months before admission & $70(13)$ & $41(12)$ & 0.62 \\
Current chemotherapy & $11(2)$ & $21(6)$ & $<0.05$ \\
Comorbid conditions & & & \\
Diabetes mellitus & $16(3)$ & $76(22)$ & $<0.05$ \\
HIV/AIDS & $0(0)$ & $3(1)$ & $<0.05$ \\
Chronic kidney disease & $11(2)$ & $35(10)$ & $<0.05$ \\
In-hospital factors & & & \\
Length of admission, days, mean & 10.6 & 14.4 & \\
TPN during admission & $59(11)$ & $0(0)$ & $<0.05$ \\
Surgery during hospital stay & $387(72)$ & $3(1)$ & $<0.05$ \\
Bowel surgery during admission & $376(70)$ & $3(1)$ & $<0.05$ \\
Antibiotics during admission & $290(54)$ & $138(40)$ & $<0.05$ \\
Blood transfusion & $81(15)$ & $24(7)$ & $<0.05$ \\
Foley catheter & $279(52)$ & $66(19)$ & $<0.05$ \\
Central line access & $81(15)$ & $28(8)$ & $<0.05$ \\
Requiring ICU admission & $11(2)$ & $3(1)$ & 0.17 \\
Ventilation & $0(0)$ & $3(1)$ & $<0.05$ \\
\hline
\end{tabular}

Data presented as $n(\%)$ unless otherwise indicated. IBD Inflammatory bowel disease; ICU Intensive care unit; TPN Total parenteral nutrition

In our study, we identified a very small subgroup of IBD patients who developed colonization or infection with ARO after hospital admission. Causes for nosocomial ARO acquisitions in the IBD inpatients are unclear, although previous literature has identified possible risk factors in the general hospitalized population (22). For example, well-described risk factors for MRSA acquisition in hospitalized patients include patient-to-patient transmission, HIV, chronic renal disease and antibiotic use $(23,24)$. However, none of these latter factors were shown to have significant correlation with the few cases of $\mathrm{ARO}$ colonization or infection acquired by our hospitalized IBD patients. Because of the few cases of ARO colonization or infection that incurred in the hospital, our study may have been underpowered to detect risk factors.

Our study also showed that, during hospitalization, infections with ARO in IBD patients occurred approximately one week sooner among those already colonized with the same $\mathrm{ARO}$ on admission than those not colonized (12 versus 20 days for MRSA; seven versus 16 days for ESBL). Although this data set is small, it can be argued that colonized IBD patients have a higher risk of infection than noncolonized IBD patients; and an excessive length of stay (past seven to 12 days) puts IBD patients, particularly those who were colonized on admission, at a higher risk for ARO infections. These findings substantiate in the IBD population previous observations that colonization by an ARO such as MRSA increases the risk of subsequent infection (25). Further studies are needed to elucidate the factors and mechanisms during hospitalization that precipitate the transition from colonization to infection.

The primary limitation of the present study was that it involved data from a single tertiary IBD centre, which may introduce referral bias and raises concerns of generalizability. IBD patients from referral centres often have more severe disease resulting in more frequent health care encounters, hospitalizations, surgeries, and use of immunosuppressive drugs and antibiotics. Given these factors, we would have expected that the prevalence of ARO colonization in our patient population would be higher than that of the general IBD population. Therefore, our finding that the risk of MRSA is consistently low in
TABLE 4

Patients with antimicrobial-resistant organism colonization and acquisition

\begin{tabular}{lcc}
\hline & \multicolumn{2}{c}{ Inpatient group } \\
\cline { 2 - 3 } & IBD $(\mathbf{n}=\mathbf{5 3 7})$ & Non-IBD $(\mathbf{n}=\mathbf{3 4 5})$ \\
\hline Colonized on admission & 5 & 4 \\
MRSA & 22 & 19 \\
ESBL & 1 & 0 \\
VRE & 2 & \\
Acquiring infection/colonization during admission & 1 \\
MRSA & 2 & 3 \\
ESBL & 9 & 0 \\
VRE & 0 &
\end{tabular}

Data presented as $n$. ESBL Extended-spectrum beta-lactamase-producing Enterobacteriaceae; IBD Inflammatory bowel disease; MRSA Methicillinresistant Staphylococcus aureus; VRE Vancomycin-resistant Enterococcus species

\section{TABLE 5}

Time to infection and colonization during admission for inflammatory bowel disease patients

\begin{tabular}{lccc}
\hline & \multicolumn{3}{c}{ Infection } \\
\cline { 2 - 4 } & MRSA & ESBL & VRE \\
\hline Patients colonized on admission & & & \\
$\quad$ Time to infection, days, mean & 12.3 & 7 & - \\
Patients not colonized on admission & & & \\
$\quad$ Time to colonization, days, mean & 35 & 6.5 & - \\
$\quad$ Time to infection, days, mean & 20 & 16.5 & - \\
\hline
\end{tabular}

ESBL Extended-spectrum beta-lactamase-producing Enterobacteriaceae; MRSA Methicillin-resistant Staphylococcus aureus; VRE Vancomycin-resistant Enterococcus species

both our inpatient and clinic-based IBD populations is reassuring. However, the higher rates of ESBL in the IBD population, which has a high prevalence of antibiotic use, reaffirms that we cannot be complacent and must exercise judicious use of antibiotics for the treatment of IBD and its complications. We should also be cautious in generalizing the low rates of ARO at our institution to other hospitals throughout Canada. Our rates of MRSA appear to be lower than that reported in the CANWARD study and may reflect effective local infection control practices.

Although we demonstrated a similar prevalence of ARO colonization between IBD and non-IBD populations, it is important for health care providers to remain vigilant regarding the potential risks for MRSA and ESBL acquisition in the hospital setting. In our study, we validated previous results that risk factors for ARO colonization in hospitalized patients include chronic renal disease and antibiotic use on admission. Furthermore, we demonstrated that IBD patients who were ARO colonizers before hospitalization developed an ARO infection sooner than their noncolonized counterparts. Given the associated cost, morbidity and mortality associated with ARO infection, future studies should focus on further elucidation of the mechanisms of $\mathrm{ARO}$ acquisition to develop novel strategies to reduce such infections. Additionally, continued efforts should be made to shorten overall length of hospitalization to avert both de novo colonization and development of infection.

ACKNOWLEDGEMENTS: This study would not have been possible without the generosity of Brian Kemp of Oxoid Inc (Nepean, Ontario), who provided all of the culture media and related supplies, and Susan Finn of StarPlex Scientific, Toronto, Ontario, who donated the swabs for specimen collection. The authors also thank Aviva Rostas, Nathan Kreiswirth, Kenzo Saito, Jessica Thom and Pirasanya Gnanasuntharam for their invaluable contributions during the study. 


\section{REFERENCES}

1. Fridkin SK, Hageman JC, Morrison M, et al. Methicillin-resistant Staphylococcus aureus disease in three communities. N Engl J Med 2005;352:1436-44.

2. Zhanel GG. Antimicrobial susceptibility of 15,644 pathogens from Canadian hospitals: Results of the CANWARD 2007-2009 study. Diag Microbiol Infect Dis 2011;69:291-306.

3. Nguyen GC, Patel H, Chong RY. Increased prevalence of and associated mortality With methicillin-resistant Staphylococcus aureus among hospitalized IBD patients. Am J Gastroenterol 2009; 105:371-7.

4. Lucet J-C. Prevalence and risk factors for carriage of methicillinresistant Staphylococcus aureus at admission to the intensive care unit - results of a multicenter study. Arch Int Med 2003;163:181.

5. Klein E, Smith DL, Laxminarayan R. Hospitalizations and deaths caused by methicillin-resistant Staphylococcus aureus, United States, 1999-2005. Emerg Infect Dis 2007;13:1840-6.

6. Safdar N, Maki DG. The commonality of risk factors for nosocomial colonization and infection with antimicrobial-resistant Staphylococcus aureus, enterococcus, Gram-negative bacilli, Clostridium difficile, and Candida. Ann Intern Med 2002;136:834-44.

7. Ananthakrishnan AN, McGinley EL. Infection-related hospitalizations are associated with increased mortality in patients with inflammatory bowel diseases. J Crohns Colitis 2013;7:107-12.

8. Bewtra M, Su C, Lewis JD. Trends in hospitalization rates for inflammatory bowel disease in the United States. Clin Gastroenterol Hepatol 2007;5:597-601.e1.

9. Munkholm P, Langholz E, Davidsen M, Binder V. Disease activity courses in a regional cohort of Crohn's disease patients. Scand J Gastroenterol 1995;30:699-706.

10. Nguyen GC, Nugent Z, Shaw S, Bernstein CN. Outcomes of patients with Crohn's disease improved From 1988 to 2008 and were associated with increased specialist care. Gastroenterology 2011;141:90-7.

11. Lichtenstein GR, Rutgeerts P, Sandborn WJ, et al. A pooled analysis of infections, malignancy, and mortality in infliximab- and immunomodulator-treated adult patients with inflammatory bowel disease. Am J Gastroenterol 2012;107:1051-63.

12. Afif W, Loftus EV Jr. Safety profile of IBD therapeutics: Infectious risks. Gastroenterol Clin North Am 2009;38:691-709.

13. Nguyen GC, Kaplan GG, Harris ML, Brant SR. A national survey of the prevalence and impact of Clostridium difficile infection among hospitalized inflammatory bowel disease patients.

Am J Gastroenterol 2008;103:1443-50.
14. Issa M, Vijayapal A, Graham MB, et al. Impact of Clostridium difficile on inflammatory bowel disease. Clin Gastroenterol Hepatol 2007;5:345-51.

15. Nguyen GC, Leung W, Weizman AV. Increased risk of vancomycinresistant enterococcus (VRE) infection among patients hospitalized for inflammatory bowel disease in the United States. Inflamm Bowel Dis 2011;17:1338-42.

16. McLaughlin SD, Clark SK, et al. Extended spectrum betalactamase-producing bacteria and Clostridium difficile in patients with pouchitis. Aliment Pharmacol Ther 2010;32:664-9.

17. Leung W, Malhi G, Willey BM, et al. Prevalence and predictors of MRSA, ESBL, and VRE colonization in the ambulatory IBD population. J Crohn's Colitis 2012;7:743-9.

18. Longobardi T, Bernstein CN. Health care resource utilization in inflammatory bowel disease. Clin Gastroenterol Hepatol 2006;4:731-43.

19. Zhanel GG, DeCorby M, Adam H, et al. Prevalence of antimicrobial-resistant pathogens in Canadian hospitals: Results of the Canadian Ward Surveillance Study (CANWARD 2008). Antimicrob Agents Chemother 2010;54:4684-93.

20. Biedenbach DJ, Moet GJ, Jones RN. Occurrence and antimicrobial resistance pattern comparisons among bloodstream infection isolates from the SENTRY Antimicrobial Surveillance Program (1997-2002). Diagn Microbiol Infect Dis 2004;50:59-69.

21. Wu H-S, Kuo S-C, Chen L-Y, et al. Comparison between patients under hemodialysis with community-onset bacteremia caused by community-associated and healthcare-associated methicillinresistant Staphylococcus aureus strains. J Microbiol Immunol Infect 2013;46:96-103.

22. Hidron AI. Risk factors for colonization with methicillin-resistant Staphylococcus aureus (MRSA) in patients admitted to an urban hospital: Emergence of community-associated MRSA nasal carriage. Clin Infect Dis 2005;41:159-66.

23. Mathews WC, Caperna JC, Barber RE, et al. Incidence of and risk factors for clinically significant methicillin-resistant Staphylococcus aureus infection in a cohort of HIV-infected adults. J Acquired Immune Def Synd 2005;40:155-60.

24. Shorr AF. Epidemiology of staphylococcal resistance. Clin Infect Dis 2007;45(Suppl 3):S171-S176.

25. Davis KA, Stewart JJ, Crouch HK, Florez CE, Hospenthal DR. Methicillin-resistant Staphylococcus aureus (MRSA) nares colonization at hospital admission and its effect on subsequent MRSA infection. Clin Infect Dis 2004;39:776-82. 


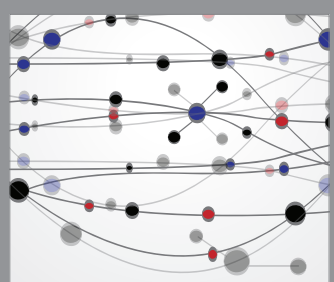

The Scientific World Journal
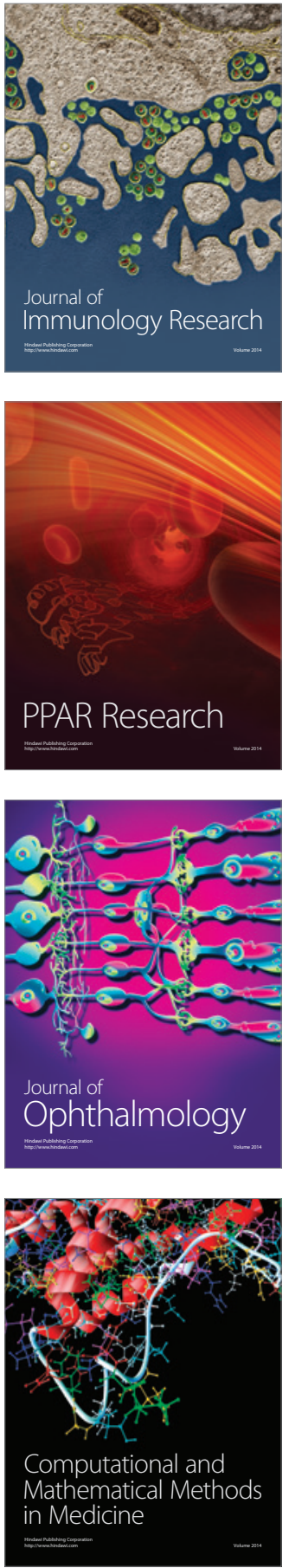

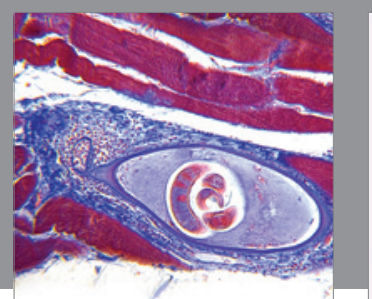

Gastroenterology Research and Practice

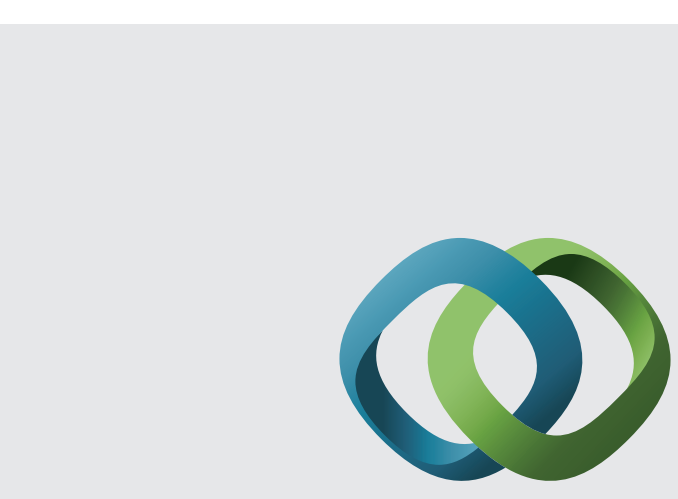

\section{Hindawi}

Submit your manuscripts at

http://www.hindawi.com
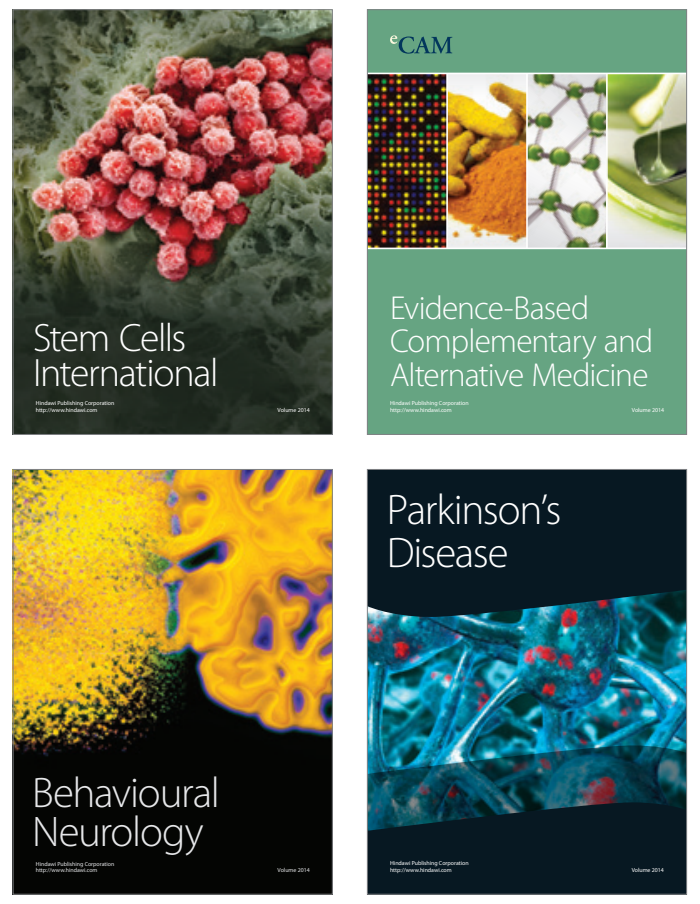
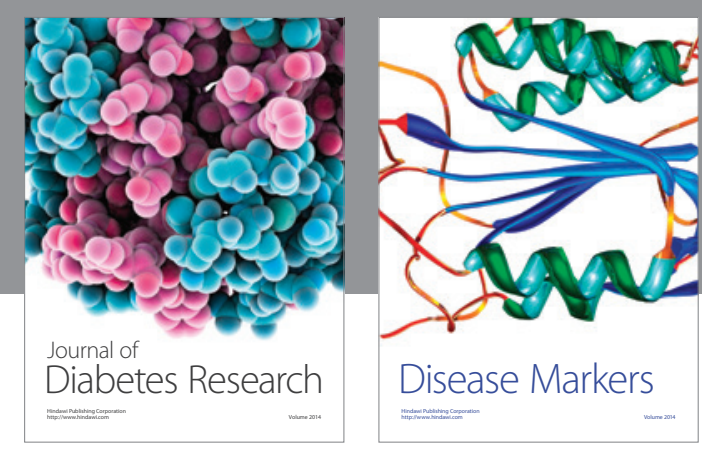

Disease Markers
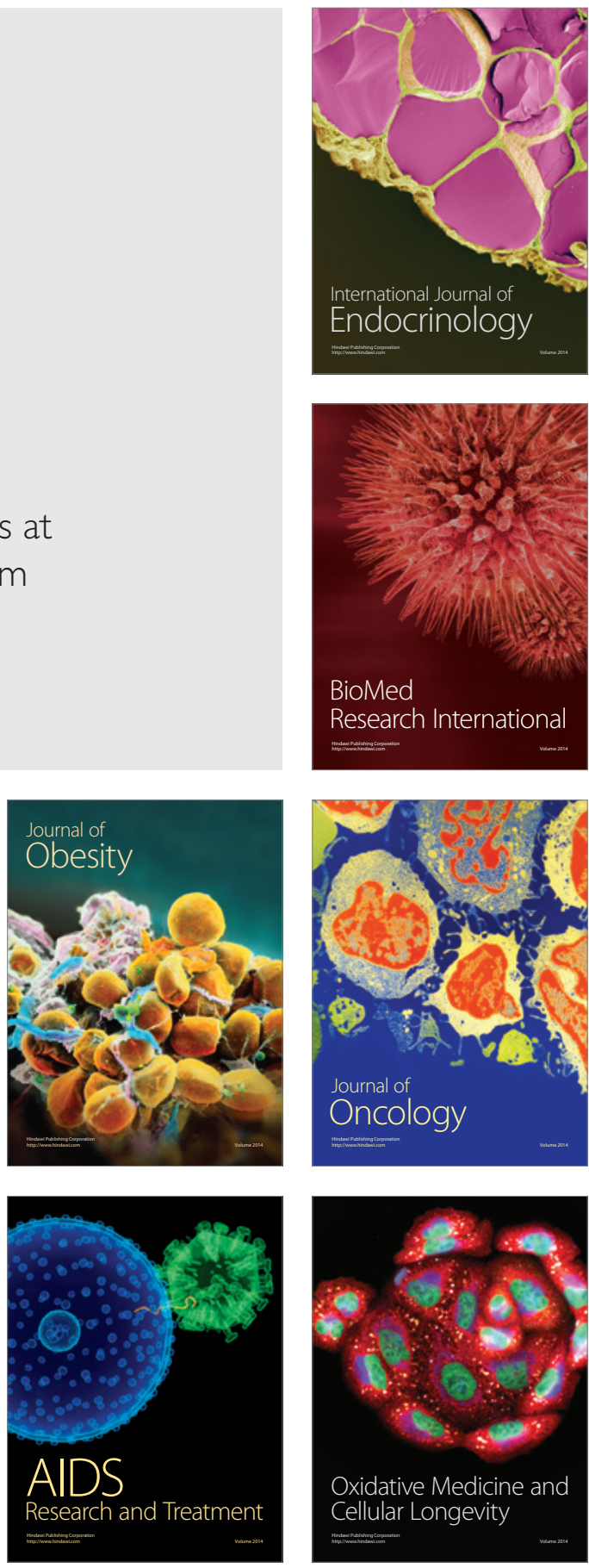